
online version of the article only. Distribution for non-commercial purposes only.

\title{
From Acute Pancreatitis to Stage IV Pancreatic Cancer in 12 Weeks
}

\author{
Chijioke Enweluzo $^{a} \quad$ Simanta Dutta ${ }^{a} \quad$ Fahad Aziz $^{a} \quad$ Stephen Lenfest ${ }^{b}$ \\ ${ }^{a}$ Section on Hospital Medicine, Department of Internal Medicine, and ${ }^{b}$ Department of \\ Pathology, Wake Forest School of Medicine, Winston Salem, N.C., USA
}

\section{Key Words}

Pancreatic cancer - Acute pancreatitis · Pancreatic cystic neoplasms

\begin{abstract}
Pancreatic cancer is well known to be an aggressive and highly malignant condition with varied ways of presentation. Pancreatic cystic neoplasms are very uncommon causes of pancreatic malignancy and can often be ignored or missed, especially in the early stages. We present the case of a 49-year-old Caucasian male with no past medical history presenting to an outside facility with sudden epigastric pain that was eventually diagnosed as acute pancreatitis. On transfer to our facility, he was eventually found to have metastatic malignant mucinous cystic pancreatic neoplasm. Barely 12 weeks after his initial presentation and following an aggressive hospital course, he passed away.

(c) 2013 S. Karger AG, Basel
\end{abstract}

\section{Introduction}

Pancreatic cancer is well known to be an aggressive and highly malignant condition with varied ways of presentation. Pancreatic cystic neoplasms (PCNs) are very uncommon causes of pancreatic malignancy and are often ignored or missed, especially in the early stages. They can be considered as confusing diagnostic problems that are being encountered with greater frequency, especially with modern and advanced imagining technology $[1,2]$. PCNs account for $<10 \%$ of all pancreatic malignancies, but for up to $30 \%$ of pancreatic resections performed, and they encompass a spectrum of benign, premalignant (borderline) and malignant lesions [1-3]. Although relatively rare, it is important to distinguish cystic neoplasms from the far more prevalent entity of benign pseudocysts, which are usually associated with a clinical history and radiographic evidence of pancreatitis. Cystic neoplasms of the pancreas can be classified into four major categories as discussed below [3]. These categories possess 
varying potentials for malignancy and prognostic outcomes, hence the need for proper diagnosis.

\section{Clinical Course}

We bring to light our experience with a 49-year-old male with no past medical history who was initially diagnosed with acute pancreatitis without any obvious cause at an outside facility after he presented with sudden epigastric pain. He was initially treated conservatively and subsequently discharged home. However, following discharge, his symptoms persisted. Two weeks later, he was readmitted to the outside facility where a laparoscopic cholecystectomy was performed for cholelithiasis that was presumed to be the cause of his persistent symptoms. At the same time he was also found to have a pancreatic pseudocyst on CT of the abdomen/pelvis (fig. 1, fig. 2, fig. 3). He was subsequently seen by gastroenterology at our hospital, where he had a EUS-FNA biopsy of the pancreatic pseudocyst, and was discharged home. However, on discharge, he started experiencing unremitting daily fevers $\left(101-104^{\circ} \mathrm{F}\right)$, nausea and diffuse abdominal pain. At this time he described some weight loss that he attributed to a loss of appetite. He presented to the emergency department as his symptoms continued to worsen.

On presentation, he was found to be in painful distress. Vitals were significant for fever with temperature at $101.7^{\circ} \mathrm{F}$. Physical examination revealed a moderately distended and tender abdomen with guarding in the epigastrium and left lower quadrant. Labs showed a hemoglobin of $11.4 \mathrm{~g} / \mathrm{dl}$, a white blood cell count of $10.2 \times 10^{3} / \mu \mathrm{l}$, AST of $85 \mathrm{U} / \mathrm{l}$, ALT of $119 \mathrm{U} / \mathrm{l}$ and alkaline phosphate of $136 \mathrm{U} / \mathrm{l}$. Lipase at the time was found to be $85 \mathrm{U} / \mathrm{l}$. He was admitted and treated conservatively for presumptive persistent pancreatitis. A CT scan of the abdomen/pelvis with contrast revealed sequela of pancreatitis with complex peri-pancreatic fluid and likely necrosis involving the distal pancreatic body and tail. A fluid level was also identified, raising concern for debris/necrotic tissue versus blood products secondary to recent bleeding into these collections. Multiple ill-defined hypoattenuating lesions scattered throughout the hepatic parenchyma were also identified, although they were considered too small to characterize (fig. 1).

These findings raised concern for infected pseudocyst. The patient was placed on Vancomycin and Zosyn from admission without any relief of his symptoms. Subsequently, he was placed on Imipenem, still without much success. He continued to experience worsening fevers, respiratory distress, abdominal pain and distension. Given these worsening symptoms, he was taken to the operating room for an exploratory laparotomy and possible pancreatic necrosectomy. Upon exploration of the abdomen, a PCN was found, with micrometastases to the liver and small bowel mesentery. Multiple biopsies were taken intraoperatively. On pathological examination, the tumor was found to be composed of highly pleomorphic, discohesive cells with eosinophilic cytoplasm and frequent multinucleated giant cells (fig. 4). The tumor was also positive for epithelial membrane antigen, confirming the epithelial nature of the malignant cells. Cytokeratin 7 and 20 markers were found to be negative (fig. 5). These findings were consistent with metastatic malignant mucinous cystic neoplasm of the pancreas. 


\section{Discussion}

Pancreatic cysts are mostly diagnosed incidentally from imaging. They range from inflammatory pseudocysts that arise from acute or recurrent pancreatitis to PCNs that possess varying malignant potential. Cystic neoplasms are a broad group with various subtypes that are responsible for about $1 \%$ of pancreatic cancers [1]. Presenting symptoms and signs are nonspecific and are no basis for diagnosis. In most cases, they remain asymptomatic until they are incidentally discovered or up to very late stages, especially in malignant subtypes. There are different subtypes of PCNs [1-3]. Serous cyst adenomas are mostly found in middle-aged females. They are typically benign, multicystic lesions formed by glycogen-rich, periodic acid-Schiff-positive epithelial cells with a central scar that can be found throughout the pancreas. Abdominal discomfort is the major presenting feature and is mainly secondary to expanding tumor size. Symptoms like jaundice and weight loss are rare. Cyst fluid analysis of serous cyst adenomas may reveal an elevated CA-19 level. Mucinous cyst adenomas are a second subtype and just like their serous counterparts are common among middle-aged females, with a higher potential for malignancy. On imaging they may be seen as one or more cysts with thick, irregular walls along the body and/or tail of the pancreas. Cystic fluid analysis of mucinous cystadenomas reveals a positive mucin stain with an elevated CEA level. A positive cytology is also not uncommon. Jaundice and weight loss are more common symptoms here. Intrapapillary mucinous neoplasia is a third subtype and unlike the subtypes discussed earlier, it is more common in middle-aged and older males. It also has a high malignant potential. Imaging here reveals pancreatic duct dilatation with papillary projections. Lastly, papillary cystic neoplasms are a rare form of cystic neoplasms found in young females. They have a malignant potential, are locally aggressive and can be found along the body and/or tail of the gland.

The most important factor to keep in mind with cystic neoplasms is the need for proper diagnosis to differentiate between subtypes. This will help assess the potential for malignancy and hence guide appropriate therapy. According to Grobmyer et al. [4] the risk of malignancy is directly proportional to the size of the cysts, especially in mucinous subtypes. Asymptomatic cysts that are $<5 \mathrm{~cm}$ at their widest diameter without concerning features such as nodularity and/or calcifications can be serially monitored at least yearly with imaging. EUS-FNA is usually added to obtain cystic fluid for analysis and cytology, thereby enhancing diagnostic accuracy [5]. CEA is the most accurate tumor marker used in diagnosing PCNs [6]. Molecular markers and cystic DNA are other methods that can be employed in proper diagnosis. In a prospective, multicenter study, Khalid et al. [7, 8] and Jones et al. [9] concluded that elevated amounts of pancreatic fluid DNA, high-amplitude mutations and specific mutation acquisition sequences are indicators of malignancy. Lesions with a high risk of malignancy and caught early are usually managed by surgery. Lesions in the pancreatic head are resected by a pancreaticoduodenectomy while those in the distal body and tail are resected by a pancreatectomy. Surveillance following surgery is unnecessary, as lesions do not generally recur. Serous cyst adenomas that are asymptomatic and do not raise concern of malignancy do not need to be resected. Cysts that are suspicious for malignancy in patients who are not surgical candidates can be managed with EUS-guided ethanol lavage with paclitaxel injection [10]. Cysts with widespread metastases have a very poor prognosis and palliative options may need to be explored.

Our experience shows the potential extent of malignant PCNs if left untreated or undiscovered. In a matter of 12 weeks, our patient went from being diagnosed with what was initially thought to be acute pancreatitis to metastatic malignant mucinous cystic pancreatic 
neoplasm that eventually cost him his life. It is our hope that our experience and this article bring to light the importance of proper diagnosis of PCNs.

\section{Disclosure Statement}

The authors declare no conflict of interest. There was no grant support.

\section{References}

1 Fernandez-del Castillo C, Tarragona J, Thayer SP, et al: Incidental pancreatic cysts: clinicopathologic characteristics and comparison with symptomatic patients. Arch Surg 2003;138:427-433; discussion 433-434.

-2 Pyke CM, van Heerden JA, Colby TV, et al: The spectrum of serous cystadenoma of the pancreas. Clinical, pathologic, and surgical aspects. Ann Surg 1992;215:132-139.

-3 Brugge WR, Lewandrowski K, Lee-Lewandrowski E, et al: Diagnosis of pancreatic cystic neoplasms: a report of the cooperative pancreatic cyst study. Gastroenterology 2004;126:1330-1336.

4 Grobmyer SR, Cance WG, Copeland EM, et al: Is there an indication for initial conservative management of pancreatic cystic lesions? J Surg Oncol 2009;100:372-374.

5 Frossard JL, Amouyal P, Amouyal G, et al: Performance of endosonography-guided fine needle aspiration and biopsy in the diagnosis of pancreatic cystic lesions. Am J Gastroenterol 2003;98:1516-1524.

6 Cizginer S, Turner B, Bilge AR, et al: Cyst fluid carcinoembryonic antigen is an accurate diagnostic marker of pancreatic mucinous cysts. Pancreas 2011;40:1024-1028.

7 Khalid A, McGrath KM, Zahid M, et al: The role of pancreatic cyst fluid molecular analysis in predicting cyst pathology. Clin Gastroenterol Hepatol 2005;3:967-973.

$>8$ Khalid A, Zahid M, Finkelstein SD, et al: Pancreatic cyst fluid DNA analysis in evaluating pancreatic cysts: a report of the PANDA study. Gastrointest Endosc 2009;69:1095-1102.

-9 Jones EC, Suen KC, Grant DR, Chan NH: Fine-needle aspiration cytology of neoplastic cysts of the pancreas. Diagn Cytopathol 1987;3:238-243.

10 Oh HC, Seo DW, Lee TY, et al: New treatment for cystic tumors of the pancreas: EUS-guided ethanol lavage with paclitaxel injection. Gastrointest Endosc 2008;67:636-642.

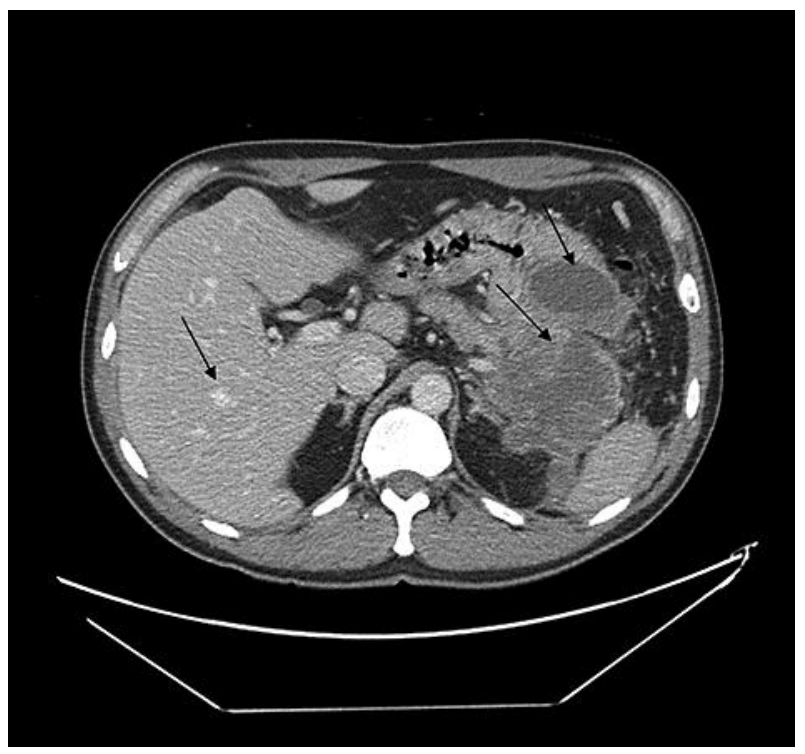

Fig. 1. CT of the abdomen/pelvis with contrast (liver and pancreas). The arrows point to areas of multiple ill-defined hypoattenuating lesions scattered throughout the hepatic parenchyma which were also identified although they were considered too small to characterize. 


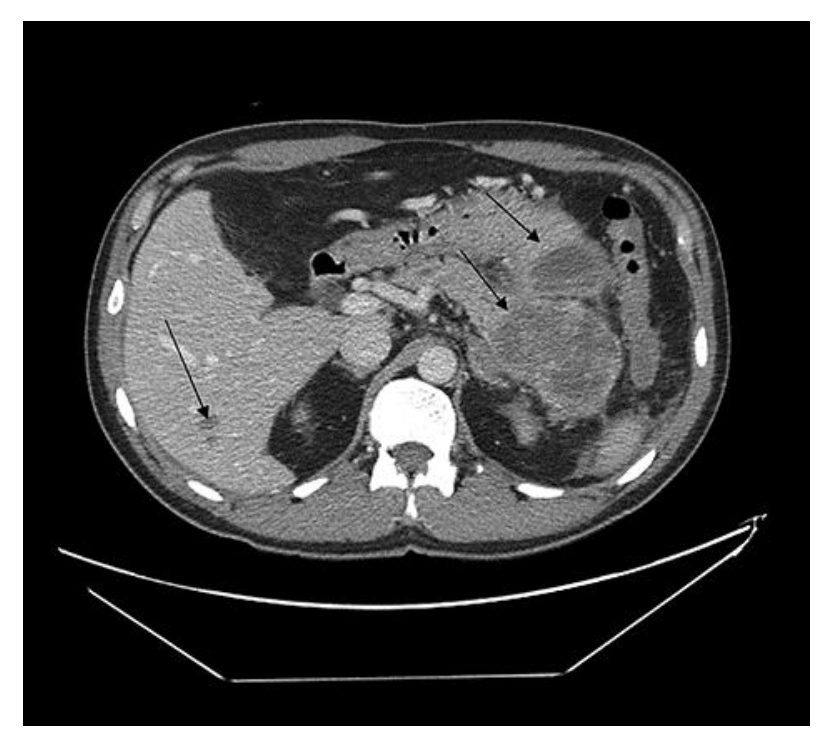

Fig. 2. CT of the abdomen/pelvis with contrast (pancreas). The arrows point to scattered areas of enhancing septation within this fluid collection. The second lobe is intimately associated with the posterior wall of the gastric fundus and likely with the gastric walls. A fluid level is seen within this portion of the fluid collection.

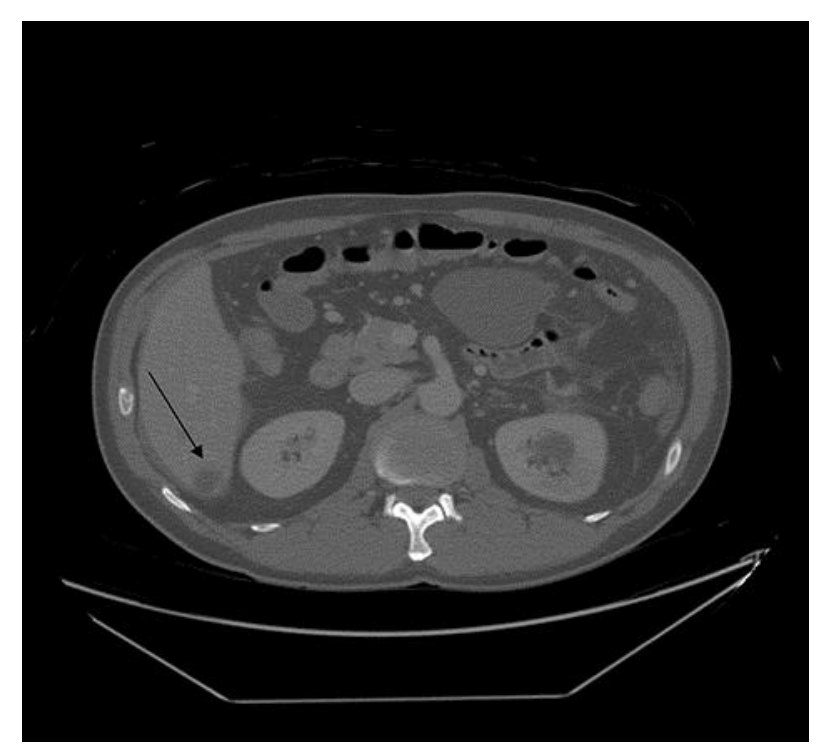

Fig. 3. CT of the abdomen/pelvis with contrast (liver). The liver measures $18.3 \mathrm{~cm}$ in its craniocaudal dimension. The arrow points to the lobulated hypoattenuating lesion within segment 6 of the liver measuring $31 \times 31 \mathrm{~mm}$ in series 2 image 56, with Hounsfield units consistent with water density. 


\begin{tabular}{|c|c|c|}
\hline \multirow{3}{*}{$\begin{array}{l}\text { Case Reports in } \\
\text { Gastroenterology }\end{array}$} & \multirow{2}{*}{\multicolumn{2}{|c|}{ Case Rep Gastroenterol 2013;7:287-292 }} \\
\hline & & \\
\hline & DOI: 10.1159/000354146 & $\begin{array}{l}\text { (c) } 2013 \text { S. Karger AG, Basel } \\
\text { www.karger.com/crg }\end{array}$ \\
\hline
\end{tabular}

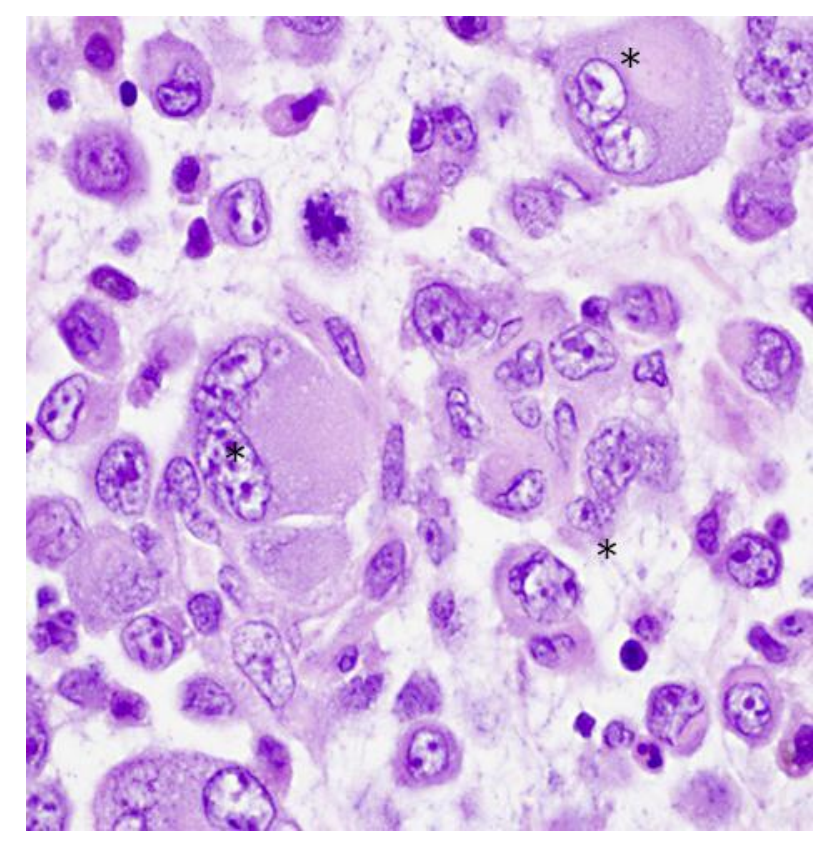

Fig. 4. Histology with hematoxylin and eosin $(\times 200)$. Representative sections of the pancreatic cyst wall and cyst contents display a tumor composed of highly pleomorphic, discohesive cells with a high nuclearto-cytoplasmic ratio (as indicated by asterisks) and eosinophilic cytoplasm in a background of mixed inflammatory infiltrate.

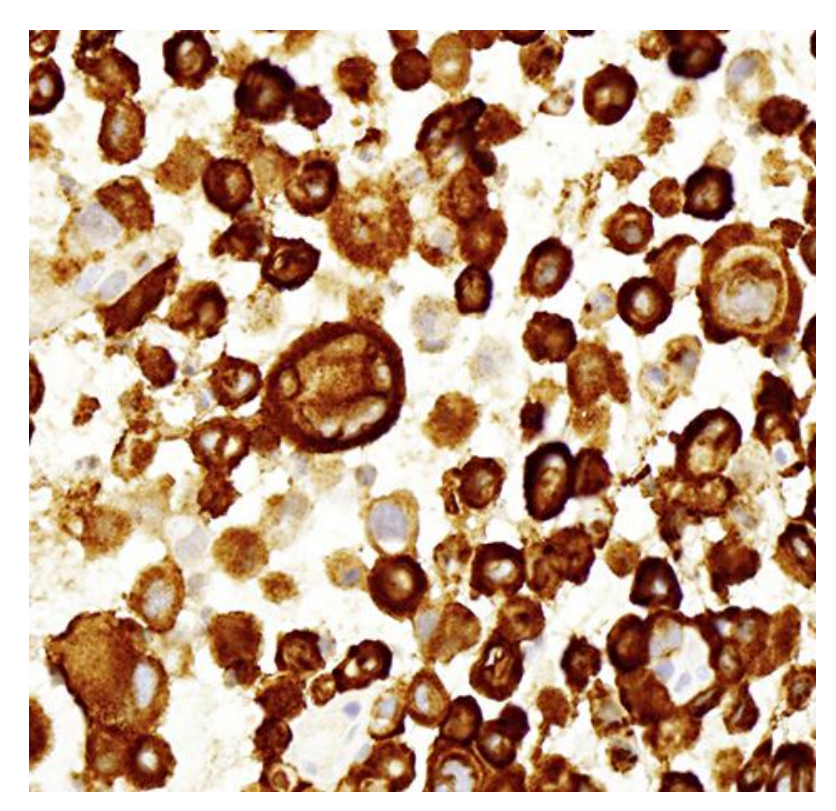

Fig. 5. Immunohistochemistry $(\times 400)$ indicating the tumor was positive for epithelial membrane antigen and negative for cytokeratin 7 and 20 . 